\title{
Sensitization to TRAIL and Radioprotective Effect of N- Coumaroyl Tyramine Diacetate from Tinospora Cordifolia Induces Apoptosis and Inhibits Neoangiogenesis to Act as an Anti-Cancer Compound.
}

\author{
Yashaswini.B, Bharathi P.Salimath* \\ Department of Studies in Biotechnology, University of Mysore, Manasagangotri, Mysore - 570 006, India.
}

\begin{abstract}
Tumor cells show differential sensitivity to cell death induced by TRAIL and also develop resistance to conventional radiation therapy along with several side effects being cytotoxic to normal cells. In this present study, we are focusing on tumor sensitization and radioprotective properties of a novel Phyto-compound isolated, purified from hexane extract and structurally identified as $N$ - Coumaroyl tyramine diacetate (N-CTD), from T.cordifolia. We have isolated a novel small molecule characterized as $N$-coumaroyl tyramine diacetate from T.cordifolia. Trypan blue exclusion assay was used to study the tumor sensitization effect of N-CTD on TRAIL-resistant cells. The flow cytometric analysis was used to analyze the efficiency of N-CTD to induce apoptosis in TRAIL-resistant cells. To assess the radioprotective effect of $\mathrm{N}$-CTD, the tumor-bearing mice were treated with $N$-CTD and irradiated with gamma radiation. The survival rate and its effect on peritoneal angiogenesis were studied. Pre-treatment with N-CTD sensitized TRAIL - resistant cells to TRAIL- mediated cell death. Flow cytometric analysis showed increased death in TRAIL- resistant cells upon combinatorial treatment. When a single dose of N-CTD combined with the sub-lethal dose of gamma radiation was initiated, the result showed a substantial decrease on peritoneal angiogenesis with increased survivability, decreased cell number and ascites volume in tumor-bearing mice treated with $\mathrm{N}$-CTD. Further, the effect was studied in terms of MVD and presence of CD31 endothelial cell marker. The results were promising to prove that N-CTD is a potential small molecule with antiangiogenic, tumor sensitization and radioprotective effect; the same can be exploited for human applications.
\end{abstract}

Keywords: Angiogenesis, Tinospora cordifolia (Willd.), TRAIL, Radiation, Ascites tumor, VEGF.

\section{Introduction}

Radiotherapy is the most routinely used conventional cancer treatment. Since, studies suggest that during this procedure, ionizing gamma radiation discharged through multiple beams towards the targeted tumor area not only kill tumor cells but also damage the surrounding normal tissues $(1,2)$. In order to avoid radiation induced damage to organs and tissues surrounding the tumor and at the same time efficiently sensitize and destroy tumor cells, small molecules derived from natural sources are in development $(3,4)$. Plant-derived small molecules have been reported to have diverse biological and pharmacological properties (5). The small phyto-molecules like withaferin-A (from Withania somnifera), apigenin, kaempferol, anthocyanidin (from Solanum nigrum L); curcumin (from Curcuma longa), and berberine (from Tinospora cordifolia), glycyrrhizin (from Glycyrrhiza glabra), diallyl disulphide, diallyl trisulphide (from Allium sativum), and capsaicin (from Capsicum annuum) have been shown to accomplish chemo-sensitizing activities both in vitro and in vivo have been considered to overcome tumor resistance to chemotherapy and as radio-sensitizers to enhance the efficacy of therapeutic radiation (6-8).

Predominantly all cytotoxic therapies are mediated by inducing apoptosis in tumor cells (9). TRAIL besides inducing apoptosis is known to have anti-tumor activity, where it showed regression of tumor growth in SCID mouse upon systemic administration. Yet, cancer cells evade TRAIL- induced cytotoxic activity due to its rapid clearance from the system and also due to death receptor dysfunction certain tumor cells evade TRAILinduced apoptosis and become resistant to death signals $(10,31)$. Plant-derived small molecules like Quercetin, curcumin, apigenin, Kaempferol, $\gamma$-Tocotrienol ( $\gamma$-T3), diosgenin, anacardic acid have been reported to sensitize resistant tumor cells to TRAIL-induced cell death (11-12).

Tinospora cordifolia is one of the noncontroversial and extensively used herbs in Ayurvedic medicine. It belongs to family Menispermaceae. It is woody climber native to Western Ghats (13). The plant has been reported to have immunomodulatory, anti-diabetic, anti- cancer and various medicinal properties and active components isolated from T. cordifolia are alkaloids, diterpenoid lactones, steroids, glycosides aliphatic compounds, polysaccharides $(14,16)$. It has been shown that the polysaccharide fraction from t. cordifolia was found to be very effective in reducing the metastatic potential of B16F-10 melanoma cells (17). The antiangiogenic and proapoptotic potential of $\mathrm{T}$. cordifolia crude extract or hexane fraction respectively has been 
reported. $(18,16)$. In our earlier studies, we have shown that the hexane fraction of T. cordifolia to be both antiangiogenic and pro-apoptotic, wherein it inhibits VEGF or MTA-1-mediated angiogenesis (19). It also inhibits transcription factors like Snail, Twist, and Slug which are associated with the epithelial-to-mesenchymal transition, a key regulatory process of metastasis (20).

The radioprotective property of Tinospora was first reported in 2002. The aqueous stem extract of Tinospora cordifolia exhibited free radical scavenging and metal chelation properties and thereby provided protection against ionizing radiation (21). We have for the first time reporting the isolation of $\mathrm{N}$-coumaroyl tyramine from T.cordifolia, earlier reports show that the compound has been isolated from A. chinense, C.fimbriata, E. racemose, I.turpethum and P. umbellatum $(22,23)$. N- trans- p-coumaroyl tyramine has been reported to exhibit potent radical scavenging effects along with inhibitory activity against cell proliferation, platelet aggregation along with anti-oxidant effect. It also shows strong suppression against phagocytic response along with anti-microbial activity (24).

Our preliminary study on the anti-angiogenic activity of hexane extract of T. cordifolia (TcHf) has shown that regular administration of $100 \mu \mathrm{g}$ for alternative days $(11.42 \mathrm{mg} / \mathrm{kg} \mathrm{b}$. w.) arrested tumor growth in EAT transplanted mice (16). Therefore, the present study was undertaken to obtain an insight into N-Coumaroyl tyramine diacetate (N-CTD) induced sensitization of TRAIL- resistant cells to TRAIL induced apoptosis, besides its radioprotective effect reflecting on survival rate.

\section{Preparation and purification of active Phyto-compound:}

\section{Materials And Methods}

The stems of T.cordifolia were collected, dried and the active compound was prepared by solvent extraction and column purification as described (25). In brief, the shade dried, coarsely powdered stems were subjected to solvent extraction based on their polarity. Extracts were evaporated by rotary evaporator. The hexane fraction having biological activity was subjected to thin layer and silica gel column chromatography, using hexane/chloroform/acetone (7:2:1) as the mobile phase. The fraction number 1 (F1) was collected and visualized under UV and extract was further analyzed for structure and molecular weight and was determined as $\mathrm{N}$-coumaroyl tyramine diacetate (N-CTD). The 10mg of the active compound was dissolved in $1 \mathrm{ml}$ DMSO and diluted further 10 times with phosphate buffered saline to make a final concentration of $1 \mu \mathrm{g} / \mu \mathrm{l}$ and used for subsequent experiments.

\section{Cell viability Assay}

In order to assess the sensitivity of the tumor cell lines, Kelly and HeLa cells to TRAIL a Trypan blue exclusion assay was done. Cells $\left(1 \times 10^{3}\right)$ were seeded onto 6 well plates and incubated overnight. The cells were either treated with N-CTD and human rTRAIL at different concentration for 12 hours or pre-treated with NCTD $(50 \mu \mathrm{g})$ for $12 \mathrm{~h}$ and then followed by treatment with different concentrations of rTRAIL at $5 \% \mathrm{CO} 2$ in $37 \mathrm{oC}$. The cells were collected by trypsinization and washed in PBS, stained with $0.2 \%$ trypan blue. The cell viability was determined by enumerating the cells using hemocytometer.

\section{Analysis of Cell Viability by Flowcytometry}

During the progression of tumor growth, determination of cell viability is a critical step when evaluating the physiological state of cells and flow cytometry provides a rapid and reliable method to quantify viable cells in a cell suspension. In Brief, HeLa cells $(1 \times 106)$ were either treated with N-CTD (50 $\square \mathrm{g} / \mathrm{ml})$ and rTRAIL $(50 \mathrm{ng} / \mathrm{ml})$ alone for 12 hours or pre-treated with N-CTD $(50 \mu \mathrm{g} / \mathrm{ml})$ for 12 hours and then followed by treatment with rTRAIL (50ng/ml) and harvested into FACS tubes. The cells were washed twice with PBS and pelleted by centrifugation at $300 \mathrm{x} g$ for 5 minutes. The cells were resuspended in $100 \mu \mathrm{l}$ of $1 \mathrm{x}$ Flowcytometry Binding Buffer (HEPES with Cacl2). A staining solution was prepared by addition of 1x binding buffer, FIT-C Annexin V, and 7-AAD solutions. The staining solution was added to the cells, mixed gently and incubated for 5 minutes at $4{ }^{\circ} \mathrm{C}$ in the dark. Data was acquired for unstained cells, single-color positive controls and stained cells on a FACS Calibur and analyzed by CELLQuest 3.2 software (both BD Biosciences). The stop count was set on the viable cells from a dot-plot of forward scatter versus 7-AAD.

\section{Radio sensitization and peritoneal angiogenesis assay.}

Source of ionizing radiation: Gamma-irradiation was given by $60 \mathrm{CO}$ irradiation at a dose of $2 \mathrm{~Gy} / \mathrm{min}$. EAT was transplanted into female Swiss albino mice. Briefly, EAT cells $(5 \times 106)$ were injected intraperitoneally (i.p) into mice (4 groups of mice, 5 in each group) and growth was recorded every day from the day of transplantation. On the 6th day after tumor inoculation, $100 \mu \mathrm{g}(2.85 \mathrm{mg} / \mathrm{kg} \mathrm{b.w})$ of N-CTD was injected (i.p). Control mice group with tumors were administered with PBS (i.p). Following 3-4 hours after injection, $2 \mathrm{~Gy}$ irradiation was given to the dorsal region of the mice. (26). 


\section{Record of Body weight, ascites volume, cell number}

The growth of the tumor in vivo was assessed by taking the weight of the animal every day. On the 14th day, one animal in each group was sacrificed, to collect the tumor cells along with ascites fluid. The cells were centrifuged and the total ascites volume measured and the number of viable cells was counted by Trypan blue exclusion method.

\section{Microvessel density counting:}

The peritoneum of the respective animals was cut open and the inner lining of the peritoneal cavity was examined for extent of neovasculature and photographed. The sections were fixed in formaldehyde and paraffin embedded tissues of the peritoneum from EAT bearing mice either treated or untreated with N-CTD were taken and $5 \mu \mathrm{m}$ sections were prepared using automatic microtome and stained with Hematoxylin and Eosin. The images were photographed using a stereo-binocular microscope with CCD camera and blood vessels were counted. The Microvessel density (MVD) count of the sections was done by Hotspot method. The mean value of 10 most vascularized areas at X 40 field was considered as MVD for a sample.

\section{CD31 Immunohistochemical staining:}

During angiogenesis, CD31 mediated endothelial cell-cell interactions are involved. Hence, to check the effect of N-CTD and ionizing radiation peritoneum sections were processed as per the protocol supplied by the manufacturer (Santa Cruz Biotechnology, CA, USA). In brief, sections were dewaxed in xylene thrice for 5 min each. The sections were rehydrated in descending concentrations of ethanol (100\% ethanol for $5 \mathrm{~min}, 95 \%$ for $2 \mathrm{~min}$ and $70 \%$ for $2 \mathrm{~min}$ ) and washed in distilled water. Antigen retrieval was done by heating the sections at $950 \mathrm{C}$ for $15 \mathrm{~min}$ in a humidified atmosphere topped with $50 \mathrm{mM}$ sodium phosphate buffer. The sections were treated with $3 \% \mathrm{H} 2 \mathrm{O} 2$ in PBS to block endogenous peroxidase activity. They were blocked in blocking serum for $30 \mathrm{~min}$ to reduce the nonspecific binding and were incubated with anti-CD 31 (PECAM-1) antibodies for 2 hrs. Following PBS wash, slides were incubated with secondary antibody (biotinylated goat anti-mouse IgG HRP) for $1 \mathrm{hr}$ at room temperature. The slides were washed in PBS for $5 \mathrm{~min}$ and antigen and antibody complex was detected using a substrate (DAB, 100 $\mathrm{l} / \mathrm{section}$ ) for $5 \mathrm{~min}$. The sections were washed thrice for $2 \mathrm{~min}$ in tap water and twice for 2 min in distilled water. Subsequently, the slides were counterstained with $2 \%$ Hematoxylin for $30 \mathrm{sec}$ and washed again in tap water thrice for $5 \mathrm{~min}$ each. The slides were dehydrated for $2 \mathrm{~min}$ each in $50 \%$ ethanol, $80 \%$ ethanol, and absolute alcohol. After xylene wash, the slides were mounted using Entellan mountant solution and the sections were scored using a stereo-binocular microscope and photographed.

\section{Results}

\section{Differential effect of N-CTD and rTRAIL on Kelly and HeLa cells}

The two cell lines (Kelly and HeLa cells) selected for the study are reported to be sensitive and resistant to TRAIL respectively. The data shown in (Fig 1A) clearly indicates that a dose response to either NCTD or rTRAIL treatment shows a dose-dependent inhibition of cell viability. The TRAIL-resistant HeLa cells, when treated in a similar manner, did not respond and show a dose-dependent inhibition of cell viability (Fig 1B). Further, pre-treatment of HeLa cells to suboptimal doses of N-CTD $(3.12 \mu \mathrm{g} / \mathrm{ml})$ for $12 \mathrm{~h}$ prior to rTRAIL for $12 \mathrm{~h}$ rendered HeLa cells sensitive to rTRAIL-induced cell death.

\section{Synergistic effect of $N$-CTD and rTRAIL on cell viability.}

Flow cytometric analysis using 7ADD showed growth inhibition in N-CTD and rTRAIL treated samples as seen in Fig 2A. Cell death was evident in N-CTD and rTRAIL alone treated cells but was significantly enhanced by $37 \%$ when treated in combination. This clearly indicates that N-CTD and rTRAIL can induce cell death individually, but a pre-treatment with N-CTD enhances the ability of rTRAIL to induce death in resistant cells.

\section{$N$-CTD enhances the radioprotective survival in vivo.}

To study the potential effect of low doses of N-CTD $(2.85 \mathrm{mg} / \mathrm{kg}$ b.w) along with low doses of radiation on the survival of EAT bearing mice, a survival study was planned. It was observed that the mice treated with $\mathrm{N}-\mathrm{CTD}$ and radiation survived for over 50days as against to mice treated only with $2 \mathrm{~Gy}$ radiation which survived for only 15 days which were similar to tumor-bearing mice without treatment. The improved inhibition of proliferation was monitored by measuring the weight of the animals every day. A decrease in body weight in animals treated with N-CTD and radiation were observed in comparison to the increased body weight of the animals treated alone with radiation and a low dose of N-CTD on tumor bearing mice. It is indicative from this data that a low dose of N-CTD is capable of inducing radioprotection and enhances survivability (Fig. 3). 


\section{$N$-CTD radiosensitizes tumor cells and inhibits peritoneal angiogenesis.}

Further, the inhibition of angiogenesis and tumor cell proliferation was evident in peritoneal angiogenesis. The body weight, ascites volume, and the total tumor cell number count were decreased (Fig 4 A, B, C). A significant decrease in blood vessel formation was observed in the peritoneum of mice treated with single dose of N-CTD along with radiation as compared with that of mice treated with N-CTD and radiation alone (Fig 4D). The effect of radiation on Average Microvessel density (MVD) count by Hotspot method and its modification by treatment with N-CTD has been depicted in (Fig 4E). From the data, it is clearly evident that administration of single dose of N-CTD $(2.85 \mathrm{mg} / \mathrm{kg} \mathrm{b.w})$ decreased MVD compared to that of the untreated tumor bearing mice. N-CTD along with radiation further decreased the MVD. The endothelial cell marker CD31 showed significant decrease in the intensity of staining and also in the number of blood vessels indicating inhibition of vascularization by N-CTD upon radiation therapy (Fig 4F)

\section{Discussion}

Conventional cancer therapy like chemotherapy and radiation therapy have several disadvantages and limitations (27). These therapies tend to induce time-dependent resistance in tumor cells (chemoresistance and radioresistance) and are cytotoxic to the surrounding normal cells with several side-effects which affect the normal functioning of organs. These therapies are also known to elicit immune-suppression and lead to physical changes which are emotionally challenging (28). Several alternative therapies have been developed to overcome the side-effects and the resistance to conventional cancer therapy. However, the results are promising but they are seldom reported.

TRAIL-mediated apoptosis is a regulatory process which is evaded by the tumor cells during proliferation and resistance to apoptosis $(29,30)$. To improve the therapeutic efficacy of any conventional therapies, they are strategically combined with death-regulating molecules like TRAIL or other novel small molecules to inhibit other tumor checkpoints like angiogenesis (31-33). Harsha Raj et.al in their study have shown that anacardic acid sensitizes TRAIL resistant Hela cells to TRAIL-induced apoptosis by regulating MAPK, NF-kB and p53. Similarly, hexane fraction (TcHf) of T.cordifolia showed pro-apoptotic activity by inducing apoptosis mediated by caspases-3 activated DNase (Thippeswamy). We in this study have reported that pre-treatment of TRAIL-resistant Hela cells by N-Coumaroyl tyramine diacetate induces TRAIL-mediated apoptosis and sensitizes the tumor cells to TRAIL-induced apoptosis. Flowcytometric analysis further proves the enhanced sensitizing effect of N-CTD to apoptosis in TRAIL-resistant cells. Understanding the mechanisms of combination therapy is necessary for the clinical development of small molecule-based drug delivery system combined with conventional therapy (34).

The mechanism of radioprotection is processed by a various parallel mechanism of action a molecule induces (35). The moment a cell is exposed to irradiation, a series of discrete reversible destructive processes are induced which can be blocked using therapeutic small molecules. The radioprotectant molecule is mechanistically classified as blockers of oxygen consumption, free radical scavengers, and DNA repair boosters, inhibitors of death signaling pathways, growth factors, blockers of radiation inflammation and chemotaxis, blockers of autocrine/paracrine pathways and keepers of genomic integrity (36).

Sachin Raj et.al have reported the inhibition of MTA-1 mediated angiogenesis by the hexane fraction of T.cordifolia. Therefore, inhibition of peritoneal angiogenesis by N-CTD which involves reduction in number of proliferating cells, decrease in the number of blood vessels and improved survivability suggest that N-CTD sensitizes tumor cells to radiation and induces radio-protection. Since the radioprotective activity by small molecules is set to associate with inhibiting autocrine pathway, our studies show that $\mathrm{N}$ - Coumaroyl tyramine diacetate induces anti-angiogenesis by inhibiting peritoneal angiogenesis in-vivo, which is mediated by VEGF; this may have a role in its radioprotective effect. In conclusion, we have shown that N-Coumaroyl tyramine diacetate sensitizes death resistant tumor cells to TRAIL-mediated apoptosis. We also demonstrate that a very low dose of N-CTD can increase survival of tumor-bearing mice exposed to whole body irradiation along with inhibition of angiogenesis in-vivo. Therefore, N-Coumaroyl tyramine diacetate with significant therapeutic properties can be exploited to treat severe maladies of cancer.

\section{Acknowledgments}

We acknowledge the financial support of Department of Biotechnology, New Delhi, India, BT/IN/German/06/BPS/2010, Department of Science and Technology, Women Scientist Scheme -A, New Delhi, India SR/WOS-A/LS-1268/2015. The authors also acknowledge the support of Prof. Yajurvedi, Coordinator, IAEC, Department of Zoology, University of Mysore for conducting animal experiments. University Grants Commission-Government of India No. f 4-1/2013SAP II and f.No. 14/4/2012(NS/PE). 


\section{Reference}

[1] Heba Sofar(2016), Cancerand Radiation Therapy advantages \& disadvantages, HEALTH

[2] Kenoki Ohuchida, Kazuhiro Mizumoto, Mitsuhiko Murakami,LiWu Qian, Norihiro Sato, EishiNagai, Kunio Matsumoto, Toshikazu Nakamura and Masao Tanaka (2004), Radiation to Stromal Fibroblasts Increases Invasiveness of Pancreatic Cancer Cells through Tumor-Stromal Interactions.Cancer Res May 1 (64) (9) 3215-3222; DOI: 10.1158/0008-5472.CAN-03-2464

[3] Baskar R, Lee KA, Yeo R, Yeoh KW (2012). Cancer and Radiation Therapy: Current Advances and Future Directions. Int J Med Sci; 9(3):193-199. doi:10.7150/ijms.3635.

[4] Charu Sharma, Bassem Sadek, Sameer N. Goyal, Satyesh Sinha, Mohammad Amjad Kamal, and Shreesh Ojha, (2015) "Small Molecules from Nature Targeting G-Protein Coupled Cannabinoid Receptors: Potential Leads for Drug Discovery and Development," Evidence-Based Complementary and Alternative Medicine, vol., Article ID 238482, 26 pages, 2015. doi:10.1155/2015/238482.

[5] Shivalika Pathania, Sai Mukund Ramakrishnan, Ganesh Bagler, (2015) Phytochemica: a platform to explore phytochemicals of medicinal plants. Database (Oxford) 2015: bav075.

[6] N. Rajendra Prasad, Ganesan Muthusamy, Mohana Shanmugam and Suresh V. Ambudkar (2016), South Asian Medicinal Compounds as Modulators of Resistance to Chemotherapy and Radiotherapy. Cancers, 8, 32; doi: 10.3390/cancers8030032.

[7] Ram V. Roy, Suman Suman, Trinath P. Das, Joe E. Luevano, and Chendil Damodaran (2013).Withaferin A, a Steroidal Lactone from Withania somnifera, Induces Mitotic Catastrophe and Growth Arrest in Prostate Cancer Cells, Journal of Natural Products 76 (10), 1909-1915.

[8] N M Reddy, Rajasekhar Reddy N, Tinospora cordifolia Chemical Constituents and Medicinal Properties: A Review, Sch. Acad. J. Pharm., 2015; 4(8): 364-369

[9] Simone Fulda and Klaus-Michael Debatin (2004) Sensitization for anticancer drug-induced apoptosis by the chemopreventive agent resveratrol, Oncogene 23, 6702-6711. doi:10.1038/sj.onc.1207630

[10] Pistritto, G., Trisciuoglio, D., Ceci, C., Garufi, A., \& D’Orazi, G. (2016). Apoptosis as anticancer mechanism: function and dysfunction of its modulators and targeted therapeutic strategies. Aging (Albany NY), 8(4), 603-619. http://doi.org/10.18632/aging.100934

[11] Stolfi, C., Pallone, F., \& Monteleone, G. (2012). Molecular Targets of TRAIL-Sensitizing Agents in Colorectal Cancer. International Journal of Molecular Sciences, 13(7), 7886-7901. http://doi.org/10.3390/ijms13077886

[12] Yi, L., Zongyuan, Y., Cheng, G., Lingyun, Z., GuiLian, Y., \& Wei, G. (2014). Quercetin enhances apoptotic effect of tumor necrosis factor-related apoptosis-inducing ligand (TRAIL) in ovarian cancer cells through reactive oxygen species (ROS) mediated CCAAT enhancer-binding protein homologous protein (CHOP)-death receptor 5 pathway. Cancer Science, $105(5)$, 520-527. http://doi.org/10.1111/cas.12395.

[13] S Shruthi, and K.K. Vijayalaxmi, 2016 "Antigenotoxic effects of a polyherbal drug septilin against the genotoxicity of cyclophosphamide in mice.," Toxicology Reports, Volume 2015 (2015), Article ID 346427, 7 pages

[14] Saha, S., \& Ghosh, S. (2012). Tinospora cordifolia: One plant, many roles. Ancient Science of Life, 31(4), 151-159. http://doi.org/10.4103/0257-7941.107344

[15] Jitendra Mittal, Madan Mohan Sharma , Amla Batra (2014) Tinospora cordifolia: a multipurpose medicinal plant- A review Journal of Medicinal Plants Studies, Year:, Volume: 2, Issue: 2.

[16] Thippeswamy G, Sheela ML, Salimath BP (2008). Octacosanol isolated from Tinospora cordifolia downregulates VEGF gene expression by inhibiting nuclear translocation of NF<kappa>B and its DNA binding activity. Eur J Pharmcol; 588:141-150.

[17] Leyon PV, Kuttan G. Inhibitory effect of a polysaccharide from Tinospora cordifolia on experimental metastasis. J Ethnopharmacol. 2004;90:233-7.

[18] Leyon PV, Kuttan G. Effect of Tinospora cordifolia on the cytokine profile of angiogenesis-induced animals. Int Immunopharmacol. 2004;4:1569-75.

[19] Sachin Raj M. Nagaraj, Yashaswini Balaraju, Niharika Shetty and Bharathi P. Salimath (2012). Metastatic events of MDA-MB-231 cells induced by angiogenic factors VEGF or MTA1 are inhibited by Tinospora cordifolia hexane fraction (tchf). IOSR Journal of Pharmacy. Volume 2 Issue 5, PP.24-30

[20] Puttananjaiah Shilpa, Yashaswini Balaraju and Bharathi P. Salimath (2015). Antimetastatic Activity of Tinospora Cordifolia Involves Inhibition of Cell Migration and Invasion Regulated By Twist and Snail Genes. IOSR Journal of Pharmacy and Biological Sciences (Volume 10, Issue 2 Ver. 1, PP 44-49.

[21] Goel HC, Kumar Prem I, Rana SV. Free radical scavenging and metal chelation by Tinospora cordifolia, a possible role in radioprotection. Indian J Exp Biol. 2002; 40:727-34.

[22] Jae B. Park, Norberta Schoene (2003), N-Caffeoyltyramine arrests growth of U937 and Jurkat cells by inhibiting protein tyrosine phosphorylation and inducing caspase-3, Cancer Letters 202 161-171.

[23] Jae B. Park and Norberta Schoene (2002), Synthesis and Characterization of N-Coumaroyltyramine as a Potent Phytochemical Which Arrests HumanTransformed Cells via Inhibiting Protein Tyrosine Kinases, Biochemical and Biophysical Research Communications 292, 1104-1110.

[24] Vetha Merlin Kumari. H, Nalini Sofia. H, Manickavasakam. K, Mohan. S, Karthikeyan. Karu,(2014) ADHATHODAI CHOORANAM : SIDDHA MEDICINE INDICATED FOR IYA ERAIPPU NOI (BRONCHIAL ASTHMA) - A REVIEW, World Journal of Pharmaceutical Research ,Volume 4, Issue 3, 802-848.

[25] Thippeswamy, G. and Bharathi P. Salimath, (2007) Induction of caspase-3 activated DNase mediated apoptosis by hexane fraction of Tinospora cordifolia in EAT cells. ENVIRONMENTAL TOXICOLOGY AND PHARMACOLOGY, 23 (2). pp. 212-220.

[26] Živilė Lukšienè (2004), Experimental evidence on possibility to radiosensitize aggressive tumors by porphyrins, Medicina (Kaunas) 2004; 40 (9): 868-874.

[27] Mondal J, Panigrahi AK and Khuda-Bukhsh AR (2014). Conventional Chemotherapy: Problems and Scope for Combined Therapies with Certain Herbal Products and Dietary Supplements. Austin J Mol \& Cell Biol.; 1(1): 10.

[28] Philip Gotwals et. al(2017), Prospects for combining targeted and conventional cancer therapy with immunotherapy, Nature Reviews Cancer, 17, 286-301

[29] Fernald, K., \& Kurokawa, M. (2013). Evading apoptosis in cancer. Trends in Cell Biology, 23(12), 620-633. http://doi.org/10.1016/j.tcb.2013.07.006

[30] Koff, J. L., Ramachandiran, S., \& Bernal-Mizrachi, L. (2015). A Time to Kill: Targeting Apoptosis in Cancer. International Journal of Molecular Sciences, 16(2), 2942-2955. http://doi.org/10.3390/ijms16022942.

[31] Stuckey, D. W., \& Shah, K. (2013). TRAIL on Trial: Preclinical advances for cancer therapy. Trends in Molecular Medicine, 19(11), 10.1016/j.molmed.2013.08.007. http://doi.org/10.1016/j.molmed.2013.08.007 
[32] Harris, S. J., Brown, J., Lopez, J., \& Yap, T. A. (2016). Immuno-oncology combinations: raising the tail of the survival curve. Cancer Biology \& Medicine, 13(2), 171-193. http://doi.org/10.20892/j.issn.2095-3941.2016.0015

[33] Tian, Z., D’Arcy, P., Wang, X., Ray, A., Tai, Y.-T., Hu, Y., Anderson, K. C. (2014). A novel small molecule inhibitor of deubiquitylating enzyme USP14 and UCHL5 induces apoptosis in multiple myeloma and overcomes bortezomib resistance. Blood, 123(5), 706-716. http://doi.org/10.1182/blood-2013-05-500033

[34] Stratford, I., Sharma, R., Plummer, R., K Stock, J., A Greenhalgh, T., Atamar, O., Wedge, S. R. (2016). Clinical Development of new Drug-Radiotherapy Combinations. Nature Reviews Clinical Oncology, 13, 627-642. DOI: 10.1038/nrclinonc.2016.79

[35] Raleigh, D. R., \& Haas-Kogan, D. A. (2013). Molecular targets and mechanisms of radiosensitization using DNA damage response pathways. Future Oncology (London, England), 9(2), 219-233. http://doi.org/10.2217/fon.12.185

[36] Koukourakis, M. I. (2012). Radiation damage and radioprotectants: new concepts in the era of molecular medicine. The British Journal of Radiology, 85(1012), 313-330. http://doi.org/10.1259/bjr/16386034

\section{FIGURES AND LEGENDS}
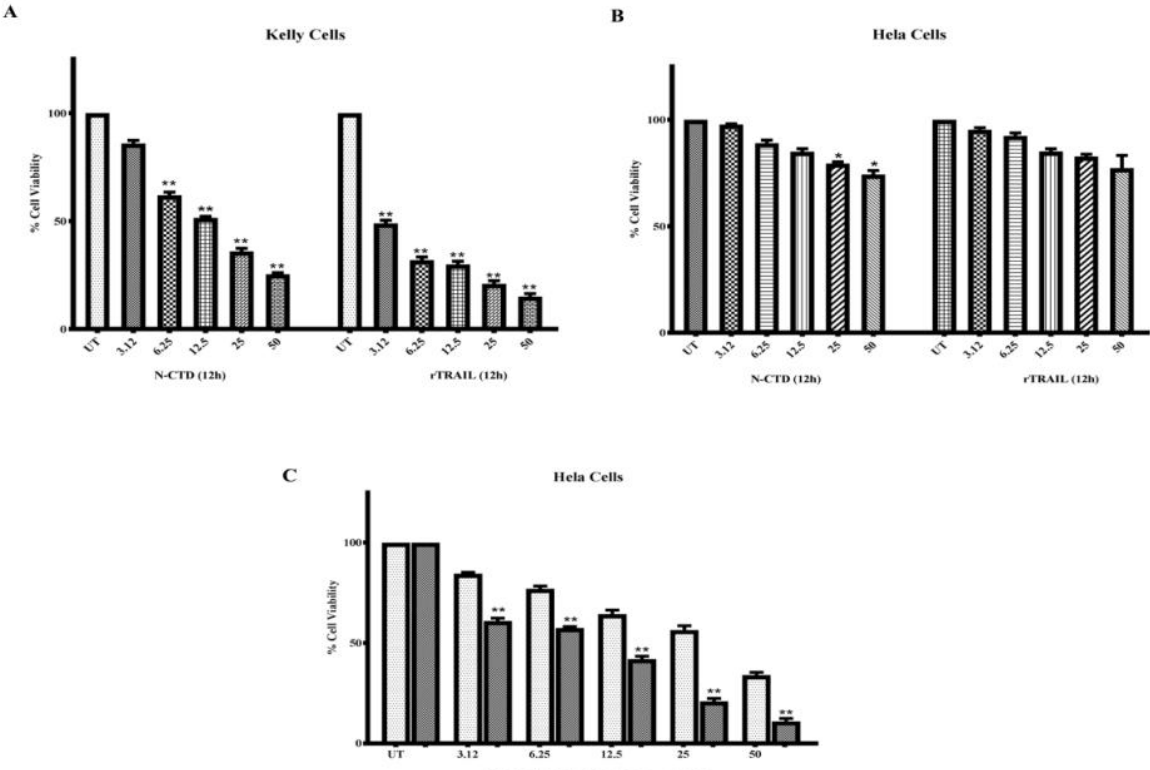

Fig1. Effect of N-CTD and rTRAIL on cell viability.

Fig1(A, B) Kelly or HeLa cells were treated with various concentrations of N-CTD $(3.12 \mu \mathrm{g}-50 \mu \mathrm{g})$ or with rTRAIL $(3.12 \mathrm{ng}-50 \mathrm{ng})$ for $12 \mathrm{~h}$. Cell viability was measured at the end of $12 \mathrm{~h}$ by trypan blue dye exclusion assay. (C), HeLa cells were sensitized with N-CTD $(50 \mu \mathrm{g})$ for $12 \mathrm{~h}$ prior to treatment with rTRAIL $(3.12 \mathrm{ng}-50 \mathrm{ng})$ for $12 \mathrm{~h}$. Cell viability was measured at the end of $24 \mathrm{~h}$. Data represent mean $\pm \mathrm{SE}$. ** $=$ significantly different from respective control; $\mathrm{P}<0.05$.

A
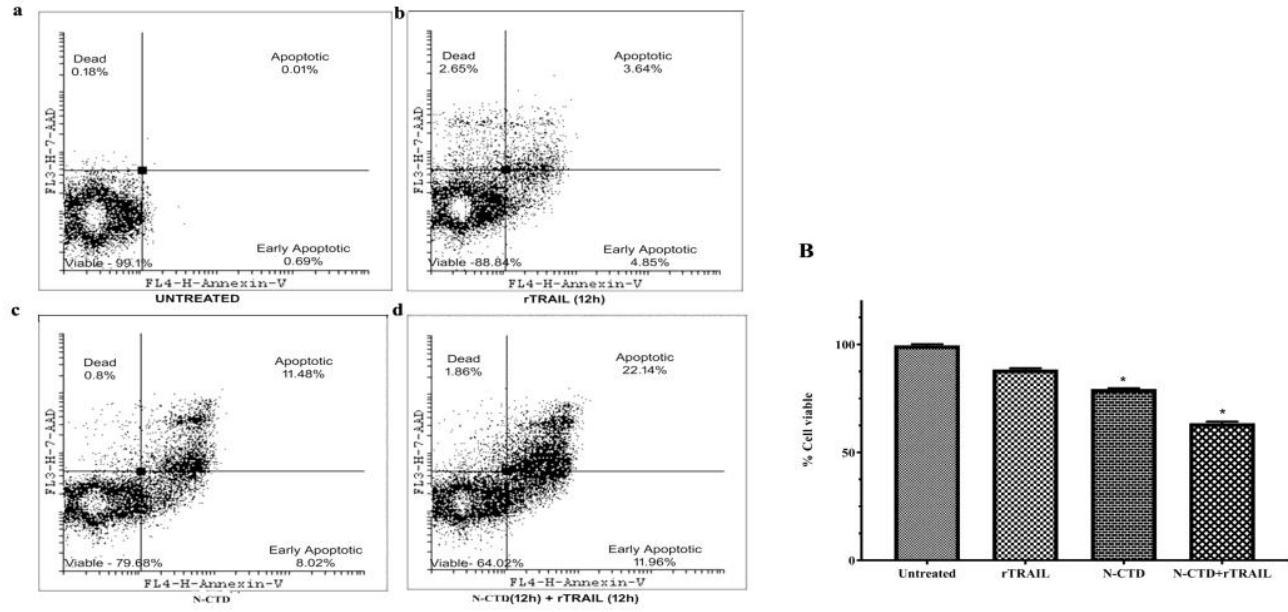
Fig 2: Flow cytometry reveals that N-CTD sensitizes TRAIL -resistant HeLa cells to rTRAIL induced cell death. Fig 2 (A) TRAIL-resistant HeLa cells were either treated with N-CTD (50 $\mu$ g) or rTRAIL (50ng) for $12 \mathrm{~h}$ and also sensitized with N-CTD $(50 \mu \mathrm{g})$ prior to treatment with rTRAIL (50ng) for another $12 \mathrm{~h}$. Cells were processed for 7AAD staining and flow cytometric analysis was performed. (B) A graphical representation of the flowcytometry data with $\%$ cell death. Data represent mean \pm SE. $* *=$ significantly different from respective control; $\mathrm{P}<0.05$.

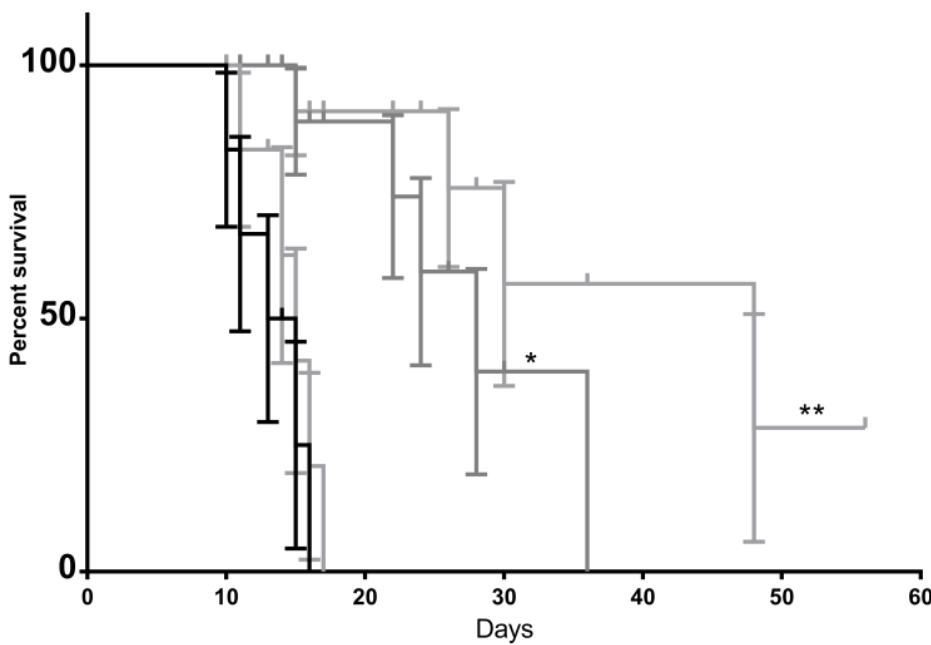

\author{
- Untreated \\ $\perp$ Radiation \\ $+\mathrm{N}-\mathrm{CTD}$ \\ - N-CTD+Radiation
}

Fig 3. Survival graph of in-vivo tumor model with N-CTD and radiation. Cumulative Survival Graph of EAT bearing mice treated with N-CTD Extract. EAT bearing mice usually have a survivability period of 12-15 days.

EAT bearing mice which were treated with N-CTD extract $(2.85 \mathrm{mg} / \mathrm{kg}$ body weight $)$ from $7^{\text {th }}$ day of tumor transplantation and irradiated with $2 \mathrm{~Gy}^{60} \mathrm{CO}$ radiation showed increased in the rate of survivability.

$\mathbf{A}$

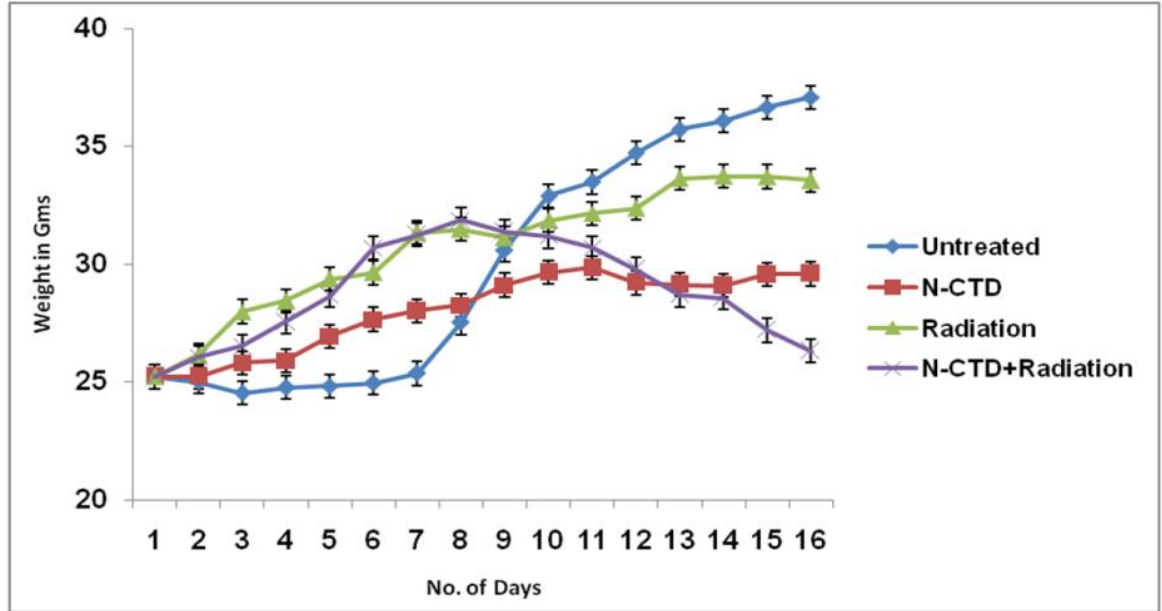

B

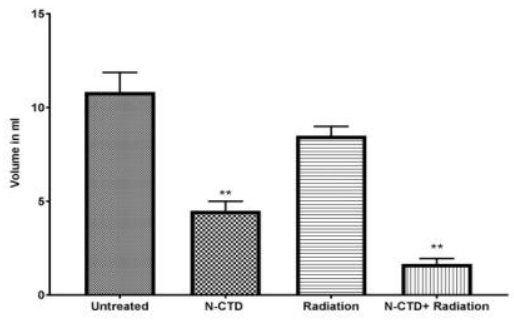

C

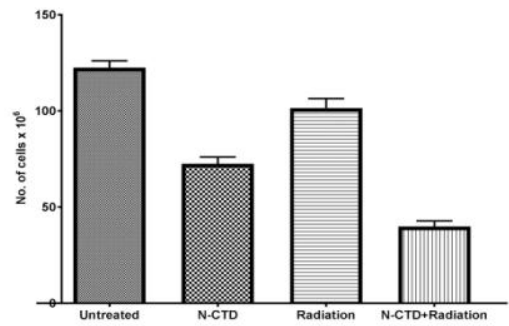


D

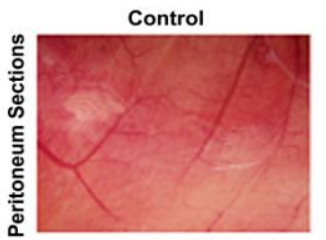

$\mathbf{E}$
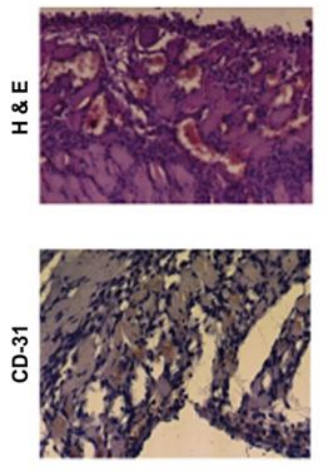

Radiation Control
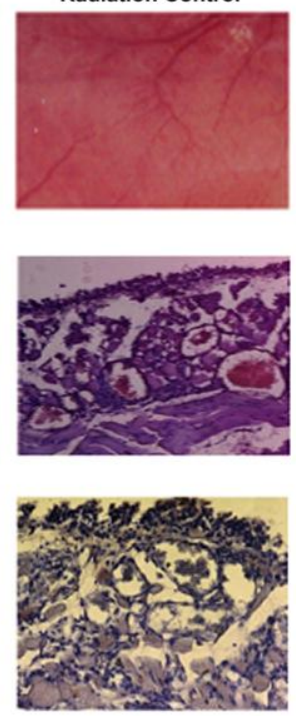

N-CTD Treated
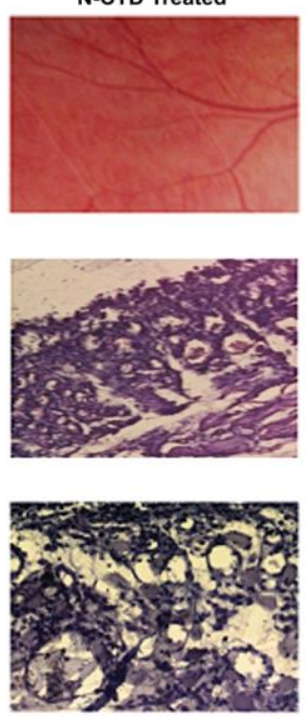

N-CTD + Radiation
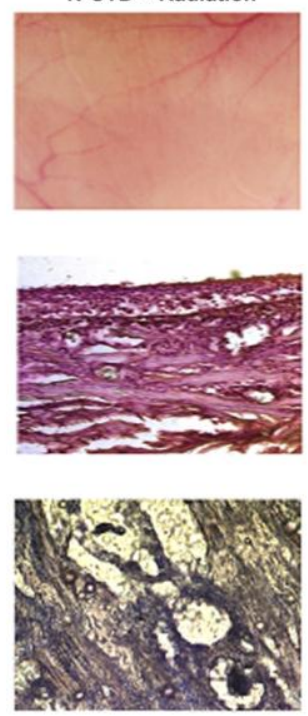

G

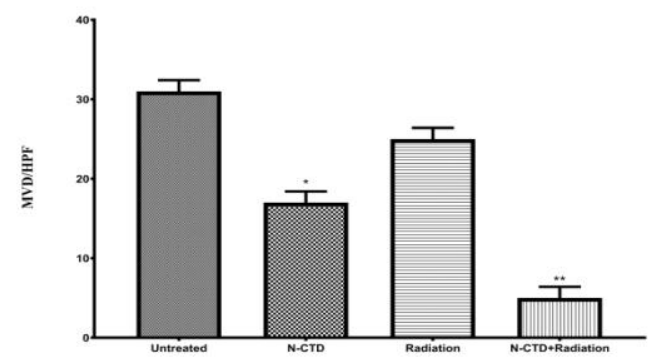

Fig 4. In vivo effect of N-CTD and Radiation treatments on EAT tumor growth.

EAT bearing mice were treated intraperitoneally with a single dose of N-CTD $(100 \mu \mathrm{g} / \mathrm{mouse}$ i.e. $2.85 \mathrm{mg} / \mathrm{kg}$ body weight) and $2 \mathrm{~Gy}$ radiation. A single mouse was sacrificed in each group on the 15 th day. A. The body weight of the mice was noted every day until the $15^{\text {th }}$ day. B. The volume of ascites formed was noted and C. Cell number was counted by trypan blue exclusion method. D. Photographs of the peritoneum were observed for the extent of vascularization. E. The peritoneum of untreated, N-CTD, radiation treated and as well as N-CTD with radiation were stained with $\mathrm{H} \& \mathrm{E}$ and observed for microvessel density. F. The sections were stained with CD31antibody. G. The microvessels were counted by Hot Spot method. The significant decrease in the microvessel density was observed in the sections from treated mice. Data represent mean $\pm \mathrm{SE}$. $*=$ significantly different from respective control; $\mathrm{P}<0.05$. 